\title{
CPNE8 as a Novel Biomarker for the Prognostic and Immunological Role by Pan-Cancer Analysis and Cerna Network of CPNE8 in Stomach Adenocarcinoma
}

Lu Lin

Guangzhou First People's Hospital https://orcid.org/0000-0001-6000-0895

Lin Lu

Guangzhou First People's Hospital

\section{Peiling Zhang}

Guangzhou First People's Hospital

Qian Peng

Guangzhou First People's Hospital

Guolong Liu

Guangzhou First People's Hospital

\section{Mingmei Guan}

Guangzhou First People's Hospital

Xiaofei Cao ( $\square$ eycaoxiaofei@scut.edu.cn )

South China University of Technology https://orcid.org/0000-0003-3330-4687

Primary research

Keywords: Pan caner, CPNE8, prognosis, immune microenvironment, ceRNA.

Posted Date: February 1st, 2022

DOI: https://doi.org/10.21203/rs.3.rs-1055878/v1

License: (a) (i) This work is licensed under a Creative Commons Attribution 4.0 International License. Read Full License 


\section{Abstract}

\section{Background}

Calcium-dependent protein copine 8 (CPNE8), a new member of Copine family, emerges important roles in malignancies. However, the expression and potential role of CPNE8 in pan cancer remain unclear.

Methods: Pan cancer analysis of CPNE8 expression in 33 types of cancers were based on data downloaded from the University of California Santa Cruz (UCSC) Xena database. The clinical significances of CPNE8 were analyzed using Cox regression analysis and Kaplan Meier survival analysis. Correlations between CPNE8 expression and tumor immune microenvironment were assessed using ESTIMATE algorithm, CIBERSORT analysis and online database TISIDB. The correlations between CPNE8 expression and tumor mutation burden (TMB), microsatellite instability (MSI) status and response to immune checkpoint inhibitors (ICls) were further explored. Moreover, competitive endogenous RNA (ceRNA) networks related to CPNE8 and its correlations with immune cell related genes were further studied in stomach adenocarcinoma (STAD).

Results: Differential expression of CPNE8 were identified in 14 types of cancers. Differential expression of CPNE8 could predict tumor stage or prognosis of various cancer patients. Pan cancer analysis indicated that CPNE8 demonstrated oncogenic role in STAD, head and Neck squamous cell carcinoma (HNSC) and esophageal carcinoma (ESCA), and tumor suppressive role in thyroid carcinoma (THCA). These four cohorts were selected for further study due to their consistent results in expression and prognostic values. Gene set enrichment analysis (GSEA) revealed that CPNE8 might regulate several key immune-related signaling pathways. Furthermore, CPNE8 expression was significantly correlated with the infiltration of different subtypes of immune cells, TMB and MSI. Moreover, PWAR5 and LIFR-AS1 could sponge miR222-3p as ceRNAs to regulate the expression of CPNE8 in STAD. In addition, CPNE8 was significantly correlated with the expression of immune cell related genes, such as CD8 T cells, macrophages, B cells and dendritic cells in STAD.

Conclusion: This study demonstrated that CPNE8 could serve as a promising biomarker associated with the prognosis and immune microenvironment in several malignancies.

\section{Introduction}

Malignancies, as the major threaten of human beings, are caused by complicated events and usually underwent multiple processes. The incidences of most cancer types are increasing yearly, such as lung cancer, breast cancer, prostate cancer, colorectal carcinoma[1-3]. For the past decades, surgery, chemotherapy and radiotherapy strategies are the primary choices in most cancer types[ 4,5$]$. Currently, the medical practices have been changed due to the development of next generation sequencing technology[6]. The emergences of targeted therapy and immunotherapy have gained a lot of attentions[7-9]. But only part of patients could benefit from targeted therapies or immune checkpoint 
inhibitors $(\mathrm{ICls})[9,10]$. Therefore, identification of novel prognostic and therapeutic biomarker is of great importance. Moreover, novel biomarkers which could be utilized in multiple cancers hold more potential.

Calcium-dependent protein copine 8 (CPNE8), belongs to copine family, is not well elucidated in carcinogenesis[11, 12]. CPNE8 was initially detected highly expressed in prostate and testis, but with low expressed in other normal tissues[13]. Currently, some bioinformatic analyses have identified aberrant expression of CPNE8 in tumors, such as breast cancer[12, 14], ovarian clear cell carcinoma[11] and cervical cancer[15]. However, expression, prognostic values and underlying mechanisms of CPNE8 in pan cancer still need to be explored. And exploring the roles of CPNE8 in other malignancies would help to identify novel diagnostic and prognostic biomarker.

The applications of immune checkpoint inhibitors (ICls), such as anti-PD1/PD-L1, have changed the treatment strategies in many types of tumors. However, unlike targeted therapies, there is lack of reliable biomarkers which could precisely predict the response to ICls[16]. Tumor mutation burden (TMB), microsatellite instability (MSI), expression of PD-L1 could partially predict the potential treatment efficacy[17]. In a word, the response rate was mainly regulated by tumor immune microenvironment[18]. The components of TME, such as tumor cells, immune cells and stoma, could manipulate the progress and response to ICls of cancers[19,20]. The correlations between CPNE8 expression and TME still need to be elucidated. Identifying novel biomarkers which could enable the predictive capabilities of current biomarkers could help us more precisely to select cancer patients who might benefit from ICls.

Non-coding RNAs (ncRNAs) are a subtype of RNAs without obvious protein coding potential, which include long non-coding RNAs (IncRNAs) and microRNAs (miRNAs)[21]. miRNA, a subtype of non-coding RNAs of about 22 nucleotides at length, could regulate the translation and stability of target mRNAs at the post-transcriptional level[21]. LncRNAs are a class of ncRNAs longer than 200 nucleotides at length. LncRNA works as a competitive endogenous RNAs (ceRNAs) in a regulatory network by sponging target miRNA to regulate mRNA expression[22]. Meanwhile, aberrant expression of IncRNAs or miRNAs were involved in the carcinogenesis and progression of malignancies[21, 22]. LncRNA RP11-396F22.1 knockdown could upregulate CPNE8 expression in cervical cancer[15]. CPNE8 was identified as one of the targeted genes of miR-375 in breast cancer[23]. Exploring IncRNA and miRNA that could regulate the expression of CPNE8 may further clarify the cause of aberrant CPNE8 expression.

Thus, in this study, we firstly investigated the expression and prognostic values of CPNE8 in pan cancer. Secondly, underlying signaling pathways of CPNE8, as well as its correlations with TME were further explored. Finally, ceRNA networks which could regulate CPNE8 expression were further studied in STAD. By studying the expression and prognostic value of CPNE8 in pan cancer, as well as InRNA-miRNA-CPNE8 network would provide potential clinical utility for novel prognostic biomarker and therapeutical biomarker.

\section{Material And Methods}


RNA-sequencing data and clinical data of pan cancer were downloaded from the University of California, Santa Cruz (UCSC) Xena database (https://xena.ucsc.edu) miRNA sequencing data was downloaded from The Cancer Genome Atlas (TCGA) database (https://portal.gdc.cancer.gov).

\section{Screening CPNE8 expression in pan cancer}

UCSC Xena database includes 33 kinds of TCGA cohorts. Differential CPNE8 expression was compared between tumors tissues and normal tissues using "limma" package in R.

\section{Clinical significance of CPNE8 in pan cancer}

Clinical data of pan cancer was downloaded from UCSC Xena database and tumor stages were retrieved. Correlations between CPNE8 expression and tumor stages were analyzed using "ggpubr" package. TISIDB, an integrated repository portal for tumor-immune system in interactions, was also used to assess the associations between CPNE8 expression and tumor stages (http://cis.hku.hk/TISIDB/index.php).

\section{Correlations between CPNE8 expression and survival}

Survival data, including survival time and survival status, was downloaded from UCSC Xena database and retrieved using "limma" package in R. The significances of CPNE8 expression in predicting overall survival (OS), disease specific survival (DSS), disease free survival (DFS) and progression free survival (PFS) were analyzed using Cox regression analysis and Kaplan-Meier survival curve analysis by using "survival", "survminer", and "forestplot" packages.

\section{ESTIMATE analysis}

ESTIMATE algorithm was used to calculate immune scores and stromal scores. The stromal score represents the presence of stroma, while the immune score represents the infiltrations of various immune cells. ESTIMATE analysis was performed using "estimate" package. And the correlations between CPNE8 expression and ESTIMATE algorithm results were assessed using Spearman correlation coefficient. And when |correlation coefficient $\mid>0.3$ and $P<0.05$ was considered as statistical significance.

\section{Tumor mutation burden (TMB) and microsatellite instability (MSI) correlation analyses}

The TMB data was downloaded from UCSC Xena database and retrieved using Perl language. The correlations between CPNE8 expression and TMB or MSI were analyzed using " $\mathrm{fmsb}$ " package in R software. And the results were demonstrated using Radar graphs.

\section{Response to ICls}

Three clinical trials data concerning the application and efficiency of anti-PD1 drugs were downloaded from GSE78220, GSE67501 and IMvigor which were involved in melanoma, human renal cell carcinoma and muscle-invasive urothelial carcinoma, respectively. Differential CPNE8 expression between response and non-response groups were assessed using "limma", "ggplot2" and "ggpubr" packages in R. 


\section{Identification of CPNE8 related miRNA}

TargetScan database邓http://www.targetscan.org/vert_80/\was used to predict the 3'UTR-binding miRNAs of CPNE8 transcripts. miRNA sequencing data for the STAD cohort were downloaded from the TCGA database. The correlation of these miRNAs with CPNE8 expression was analyzed using the "limma", "reshape2", "ggplot2" and "ggpubr" packages in R language. In addition, the prognostic value of CPNE8 plus associated miRNAs was analyzed using the "limma", "survival" and "survminer" packages in the R language.

\section{Construction of ceRNA network}

LncRNAs that could interact with miRNA-30e-5p or miRNA-222-3p were retrieved from ENCORI database 4

叉. Target IncRNAs were identified by setting the |coeffcient value| $>0.2$ and $P<0.001$ using "limma", "reshape2", "ggplot2" and "ggpubr" packages in R. Prognostic significances of these IncRNAs were analyzed using "limma", "survival" and "survminer" packages in R. Cytoscape_v3.7.2 was used to draw the ceRNA network graph.

\section{Correlations with immune cell related genes}

The correlations between CPNE8 expression and immune cell related gene expression were analyzed using "limma", "reshape2", "ggpubr", and "ggExtra" packages in STAD cohort. The gene list for each immune cell was as follows: CD19 and CD79A represent B cells; CD8A and CD8B represent CD8+ T cell; CD4 represents CD4+ T cell; NOS2, IRF5 and PTGS2 represent M1 macrophage; CD163, VSIG4 and MS4A4A represent M2 macrophage; CEACAM8, ITGAM and CCR7 represent Neutrophil; HLA-DPB1, HLADQB1, HLA-DRA, HLA-DPA1, CD1C, NRP1 and ITGAX represent Dendritic cell.

\section{Statistical analyses}

Data retrieval was processed using Perl software. All statistical analyses were conducted using $\mathrm{R}$ software (version 4.1.0). $P<0.05$ or 0.01 was defined as a significant difference.

\section{Results}

\section{Differential CPNE8 expression in pan cancer}

The UCSC Xena database was utilized to analyze the differential CPNE8 expression in pan cancer, which was consisted of 33 types of TCGA cohorts. CPNE8 expression was the highest in Acute Myeloid Leukemia (LAML) and the lowest in Brain Lower Grade Glioma (LGG) (supplementary Figure 1). Upregulated CPNE8 expression was detected in Cholangiocarcinoma ( $\mathrm{CHOL})(P<0.01)$, Esophageal carcinoma (ESCA) $(P<0.01)$, Head and Neck squamous cell carcinoma (HNSC) $(P<0.001)$, Kidney renal clear cell carcinoma (KIRC) $(P<0.001)$, Kidney renal papillary cell carcinoma (KIRP) $(P<0.05)$ and Lung squamous cell carcinoma (LUSC) $(P<0.01)$ (Figure 1A). Downregulated CPNE8 expression was detected in Bladder Urothelial Carcinoma (BLCA) $(P<0.01)$, Breast invasive carcinoma (BRCA) $(P<0.001)$, Colon 
adenocarcinoma (COAD) $(P<0.001)$, Kidney Chromophobe $(\mathrm{KICH})(P<0.001)$, Prostate adenocarcinoma (PRAD) $(P<0.001), \quad$ Rectum adenocarcinoma (READ) $(P<0.001)$, Thyroid carcinoma $($ THCA $)(P<$ $0.001)$ and Uterine Corpus Endometrial Carcinoma (UCEC) $(P<0.001)$ (Figure 1A).

\section{Clinical significance of CPNE8 in pan cancer}

Correlations between CPNE8 expression and tumor stages were also studied in pan cancer. As shown in Figure 1B, there were positive correlations between CPNE8 expression and advanced tumor stage in Skin Cutaneous Melanoma (SKCM) $(P<0.05)$ and Stomach adenocarcinoma (STAD) $(P<0.05)$. CPNE8 expression was negatively correlated with the tumor stage in BLCA $(P<0.001), \mathrm{KICH}(P<0.01)$, and THCA $(P<0.01)$. Moreover, TISIDB data also verified that higher CPNE8 expression was correlated with advanced stage in LUSC, STAD and UCEC. While lower CPNE8 expression was positively correlated with advanced tumor stage in $\mathrm{KICH}, \mathrm{THCA}$ and Uterine Carcinosarcoma (UCS) (Figure 1C).

\section{Prognostic value of CPNE8 in pan cancer}

Moreover, the roles of CPNE8 in predicting overall survival (OS), disease specific survival (DSS), disease free survival (DFS) and progression free survival (PFS) were determined using both Cox regression analysis and Kaplan Meier survival analysis.

Firstly, Cox regression analysis showed that high CPNE8 expression was risk factor for OS in LGG (HR=1.7135, $P=1.52 \mathrm{E}-05)$, STAD (HR=1.7227, $P=6.28 \mathrm{E}-05)$, LAML (HR=1.3314, $P=9.22 \mathrm{E}-05)$, UCEC $(\mathrm{HR}=1.3419, P=0.008), \mathrm{BLCA}(\mathrm{HR}=1.2052, P=0.0165), \mathrm{PAAD}(\mathrm{HR}=1.5876, P=0.0237)$, and HNSC (HR=1.2019, $P=0.0392$ ) (Table 1). While high CPNE8 was protective factor for the OS of UCS (HR=0.6103, $P=0.0346)$ (Table 1). Kaplan Meier survival curves showed that high CPNE8 expression predicted poor OS in ACC (Figure 2A, $P=0.003$ ), GBM (Figure 2B, $P=0.039$ ), HNSC (Figure 2C, $P=0.043$ ), LAML (Figure 2D, $P<0.001$ ), LGG (Figure 2E, $P=0.034$ ), and STAD (Figure 2F, $P=0.004$ ).

Prognostic values of CPNE8 expression in predicting DSS were also analyzed by Cox regression analysis and KM analysis. As shown in Table 2, high CPNE8 expression was risk factor related to DSS in STAD (HR=2.0208, $P=5.89 \mathrm{E}-05)$, UCEC (HR=1.6241, $P=7.91 \mathrm{E}-05)$, LGG (HR=1.7023, $P=8.10 \mathrm{E}-05)$ and BLCA (HR=1.2619, $P=0.0124)$. On the contrary, high CPNE8 expression was correlated with better DSS in KIRC (HR=0.7199, $P=0.0128)$ and UCS (HR=0.5911, $P=0.0330$ ) (Table 2). KM curve analysis also showed that high CPNE8 expression was correlated with poor DSS in ACC (Figure 2G, $P=0.014$ ), GBM (Figure 2H, $P=$ 0.020), LGG (Figure 2l, $P=0.044$ ), STAD (Figure 2J, $P=0.001$ ), UCEC (Figure $2 \mathrm{~K}, P=0.008$ ).

In addition, high CPNE8 expression was risk factor correlated to DFS in STAD (HR=2.3955, $P=0.0015)$ and ESCA (HR=2.0800, $P=0.0086$ ) (Table 3 ). KM curve revealed that high CPNE8 expression predicted poor DFS in STAD $(P=0.007)$ (Figure 2L).

Moreover, Cox regression analysis revealed that high CPNE8 expression was bad predictor for PFS in STAD (HR=1.7987, $P=5.11 \mathrm{E}-05)$, LGG (HR=1.3652, $P=0.0034)$, GBM (HR=1.2647, $P=0.0243)$, and BLCA $(H R=1.1922, P=0.0272)$. While highCPNE8 expression was good predictor for PFS in KIRP 
( $\mathrm{HR}=0.6367, P=0.0328)$ and THCA ( $\mathrm{HR}=0.4728, P=0.0389)$ (Table 4). KM survival analysis indicated that high CPNE8 expression was correlated with poor PFS in ACC (Figure 2M, $P=0.008$ ), GBM (Figure 2N, $P=$ 0.008 ) and STAD (Figure 20, $P<0.001$ ).

\section{Functional annotation of CPNE8}

The above results indicated that high CPNE8 expression was correlated with advanced tumor stage, poor OS, DSS, DFS and PFS in STAD. High CPNE8 expression was correlated with poor OS in HNSC. High CPNE8 expression was correlated with poor DFS in ESCA. While CPNE8 expression was a good factor in predicting PFS in THCA. Thus, GSEA was utilized to explore the potential mechanisms of CPNE8 in STAD, HNSC, ESCA and THCA. As shown in Figure 3, CPNE8 related signaling pathways were mainly enriched in Antigen processing and presentation, Cytosolic DNA pathway, Toll like receptor signaling pathway, Olfactory transduction, Calcium signaling pathway, Cell adhesion molecules cams, et al.

\section{Correlations between CPNE8 expression and tumor immune microenvironment}

GSEA results showed that the functional annotation of CPNE8 were mainly enriched in immune-related signaling pathways. Thus, we further explored the correlations between CPNE8 expression and immune microenvironment of STAD, HNSC, ESCA and THCA. Firstly, ESTIMATE algorithm was used to calculate the stromal score, immune score and ESTIMATE score. Spearman correlation coefficient analysis showed that CPNE8 expression was positively correlated with stromal score in STAD (Figure 4A, R=0.38 $P=1.8 \mathrm{e}-$ 14). Secondly, the distribution of tumor-infiltrating immune cell (TIC) abundance was analyzed using CIBERSORT analysis. As shown in Figure $4 B$ and $C$, CPNE8 expression was positively associated with the infiltration of dendritic cells (Figure $4 \mathrm{~B}, \mathrm{R}=0.38 P=3.4 \mathrm{e}-06$ ) and negatively associated with $\mathrm{T}$ cell regulatory infiltration (Figure 4C, $\mathrm{R}=-0.41 P=3.2 \mathrm{e}-07$ ) in ESCA. Moreover, TISIDB results found that CPNE8 expression was positively correlated with CD56bright natural killer cell abundance (Figure 4D, $\mathrm{R}=0.327 P=6.47 \mathrm{e}-06$ ) and negatively correlates with CD56dim natural killer cell abundance (Figure $4 \mathrm{E}$, $\mathrm{R}=-0.314 P=1.45 \mathrm{e}-05$ ) and Monocyte abundance (Figure 4F, $\mathrm{R}=-0.376 P=1.72 \mathrm{e}-07$ ) in ESCA. In STAD, CPNE8 expression was positively correlated with Macrophage abundance (Figure 4G, $R=0.322, P=2.33 e-$ 11), Mast cell abundance (Figure $4 \mathrm{H}, \mathrm{R}=0.42 \quad P<2.2 \mathrm{e}-16$ ) and Natural killer $\mathrm{T}$ cell abundance (Figure $4 \mathrm{I}$, $\mathrm{R}=0.366 P=1.57 \mathrm{e}-14)$. In THCA, CPNE8 expression was negatively correlated with CD56dim natural killer cell abundance (Figure 4J, R=-0.309 $P=1.33 \mathrm{e}-12$ ).

\section{Correlations between CPNE8 expression and TMB, MIS and response to ICls}

The above studies revealed that CPNE8 expression was closely correlated with tumor immune microenvironment. Thus, we further investigated the correlations between CPNE8 expression and immunotherapy indicators (TMB and MSI), as well as response to ICls. As shown in Figure 5A, CPNE8 expression was positively correlated with TMB in HNSC ( $\mathrm{R}=0.265, P=2.23 \mathrm{e}-09)$, THYM $(\mathrm{R}=0.331, P=0.0002)$, TGCT $(\mathrm{R}=0.206, P=0.0125)$ and $\mathrm{KIRC}(\mathrm{R}=0.1202, P=0.028)$, and negatively correlated with TMB in STAD ( $\mathrm{R}=-0.366, P=3.64 \mathrm{e}-13), \mathrm{PRAD}(\mathrm{R}=-0.230, P=3.00 \mathrm{e}-07)$, THCA $(\mathrm{R}=-0.112, P=0.013)$, and LIHC $(\mathrm{R}=-0.124, P=0.018)$. 
Correlations between CPNE8 expression and MSI showed that CPNE8 expression was positively correlated with $\mathrm{MSI}$ in BRCA ( $\mathrm{R}=0.083, P=0.007)$ and $\mathrm{READ}(\mathrm{R}=0.199, P=0.013)$. While CPNE8 expression was negatively correlated with $\mathrm{MSI}$ in STAD ( $\mathrm{R}=-0.269, P=1.15 \mathrm{e}-07)$, $\mathrm{DLBC}(\mathrm{R}=-0.490, P<0.001), \mathrm{LUAD}$ $(\mathrm{R}=-0.144, P=0.001)$, and $\mathrm{KICH}(\mathrm{R}=-0.265, P=0.032)$ (Figure $5 \mathrm{~B})$.

We next studied the correlations between CPNE8 expression and response to ICls in three cohorts. GSE78220 and GSE67501 cohorts analyses showed that there were no significant difference in CPNE8 expression between response and non response groups in melanoma (Figure $5 \mathrm{C}, P=0.76$ ) and human renal cell carcinoma (Figure 5D, $P=0.23$ ). IMvigor cohort results showed that the expression of CPNE8 was lower in response group than that in the non-response group in muscle-invasive urothelial carcinoma (Figure 5E, $P=0.0029$ ).

\section{Identification of miRNA correlated to CPNE8 in STAD}

The above results showed that CPNE8 expression was positively correlated with advanced tumor stage, poor OS, DSS, DFS and PFS in STAD. Moreover, the downstream mechanisms of CPNE8 were immune related. Thus, we further explored the upstream IncRNA-miRNA-CPNE8 network in STAD.

miRNAs that could interact with CPNE8 were retrieved using TargetScan (Figure 6A). And we identified miRNA-30e-5p and miRNA-222-3p using the following criteria: downregulated expression in STAD tumor tissues, negative correlation with CPNE8 (coefficient $>0.2, P<0.05$ ). There were negative correlations between CPNE 8 and miRNA-30e-5p (Figure 6B, $\mathrm{R}=-0.24, P=1.9 \mathrm{e}-06$ ). Kaplan Meier survival curve showed that high miRNA-30e-5p expression plus low CPNE8 expression group demonstrated better overall survival than low miRNA-30e-5p expression plus high CPNE8 expression group ( $P=0.008$, Figure $6 \mathrm{C})$. Figure 6D showed the potential binding sites between CPNE8 and miRNA-30e-5pMeanwhile, miRNA222-3p expression was negatively correlated with CPNE8 expression (Figure 6E, $\mathrm{R}=-0.23, P=9.4 \mathrm{e}-06$ ). And high miRNA-222-3p expression plus low CPNE8 expression predicted better overall survival than low miRNA-222-3p expression plus high CPNE8 expression group in STAD ( $P=0.046$, Figure 6F). Moreover, the potential binding sites between CPNE8 and miRNA-222-3p were demonstrated in Figure $6 \mathrm{G}$.

\section{Construction of ceRNA}

We next identified IncRNAs which could regulate miRNA-30e-5p or miRNA-222-3p and CPNE8 to construct a ceRNA network in STA. Potential IncRNAs that could interact with miRNA-30e-5p or miRNA-222-3p were downloaded from TargetScan. We selected IncRNA which was positively correlated with CPNE8 expression and negatively correlated with miRNA-30e-5p or miRNA-222-3p expression. As shown in Figure 7A and $B$, NORD expression was positively correlated with CPNE8 ( $R=0.1, P=0.046)$ and negatively correlated with miRNA-30e-5p (R=-0.21, $P=3.7 e-05)$.

Meanwhile, LIF-AS1 expression was positively correlated with CPNE8 (Figure 7C, R=0.43, $P=2.2 \mathrm{e}-16$ ) and negatively correlated with miRNA-222-3p (Figure 7D $\mathrm{R}=-0.6, P=5.4 \mathrm{e}-09$ ). In addition, PWAR5 expression was positively correlated with CPNE8 (Figure 7E, $\mathrm{R}=0.41, P=2.2 \mathrm{e}-16$ ) and negatively correlated with 
miRNA-222-3p (Figure 7F, $\mathrm{R}=-0.5, P=2.2 \mathrm{e}-16$ ). Given the correlations between NORD and miRNA-30e-5p or CPNE8 were weak. Thus, we constructed LIF-AS1 /miRNA-222-3p/CPNE8 and PWAR5/miRNA-2223p/CPNE8 networks in STAD.

\section{Correlations between CPNE8 expression and immune cell related genes}

We further studied the correlations between CPNE8 expression and immune cells by analyzing the correlations between CPNE8 expression and immune cell related genes. As shown in Figure 8, CPNE8 was positively correlated with $\mathrm{CD} 8$ (Figure $8 \mathrm{~A}, \mathrm{R}=0.2, P=7.2 \mathrm{e}-05$ ), IRF5 (Figure 8B, $\mathrm{R}=0.21, P=3 \mathrm{e}-05$ ), $\mathrm{PTGS} 2$ (Figure 8C, $\mathrm{R}=028, P=4.2 \mathrm{e}-08$ ), $\mathrm{CD} 163$ (Figure 8D, $\mathrm{R}=0.25, P=9.1 \mathrm{e}-07$ ), VSIG4 (Figure 8E, $\mathrm{R}=0.24, P=2.6 \mathrm{e}$ 06), MS4A4A expression (Figure 8F, $\mathrm{R}=0.28, P=7 \mathrm{e}-08$ ), ITGAM (Figure 8G, $\mathrm{R}=0.32, P=2.9 \mathrm{e}-10$ ), CCR7 (Figure 8H, $\mathrm{R}=0.22, P=2.6 \mathrm{e}-05$ ), CD1C (Figure 8I, $\mathrm{R}=0.26, P=2.1 \mathrm{e}-07$ ) and ITGAX (Figure 8J, R=0.24, $P=3.8 \mathrm{e}-06)$. These data indicated that CPNE8 expression was positively correlated with CD8,

\section{Discussion}

Using RNA-sequencing data and clinical data of 33 kinds of cancers downloaded from the UCSC Xena database, we identified upregulated CPNE8 expression in 7 TCGA cohorts (CHOL, COAD, ESCA, HNSC, KIRC, KIRP, LUSC) and downregulated CPNE8 expression in 5 TCGA cohorts (BLCA, BRCA, KICH, PRAD, READ). The clinical significances of CPNE8 expression were analyzed by assessing the correlations with tumor stage and patients' survival, including OS, DSS, DFS and PFS. We next selected TCGA cohorts with consistent results in differential expression and prognosis for further study. Pan cancer analysis showed that high CPNE8 expression was correlated with advanced tumor stage, poor OS, DSS, DFS and PFS in STAD. High CPNE8 expression was correlated with poor OS in HNSC. High CPNE8 expression was correlated with poor DFS in ESCA. While CPNE8 expression was a good factor in predicting PFS in THCA. Currently, there were limited studies concerning the roles of CPNE8 expression in carcinogenesis $[14,15$, $23,24]$. CPNE8 expression was initially identified expressed in testis and prostate[13]. Nagasawa et al. reported that knock down of CPNE8 inhibited the proliferation of clear cell carcinoma (CCC) cells and high-grade serous carcinoma (HGSC) cells[11]. Here CPNE8 expression demonstrated oncogenic roles in STAD, HNSC and ESCA. In THCA, CPNE8 could work as a tumor suppressor.

Literature searching revealed that CPNE8 worked as lipid binding protein. Reinhardt et al found that functions of CPNE8 depended on ERK activity in pankinsonian neurodegeneration[25]. However, no studies explored the working mechanism of CPNE8 in malignancies. Utilizing GSEA, we found that the underlying mechanisms concerning CPNE8 were enriched in immune-related and adhesion-related, such as antigen processing and presentation, toll like receptor signaling pathway, cell adhesion molecules cams, focal adhesion in STAD, ESCA, HNSC and THCA. Increasing studies suggested that the progression of tumors were the results of the interactions between various components of the tumor immune microenvironment (TME). TME was consisted of tumor cells, immune cells, stroma and various soluable factors. Our GSEA results revealed that CPNE8 was closely correlated to the immune-related signaling pathway. Thus, we further explored the correlations between CPNE8 expression and the tumor immune 
microenvironment of STAD, HNSC, ESCA and THCA. Firstly, ESTIMATE analysis was used to explore the correlations between CPNE8 expression and immune score, stromal score and ESTIMATE score. Here, CPNE8 expression was positively correlated with the stromal score of STAD. This result was consistent with the GSEA results in STAD, where the underlying signaling pathways were cell adhesion molecules cams and focal adhesion, which were closely related to tumor stroma.

Moreover, the correlations between CPNE8 expression and infiltrations of various immune cells were determined using CIBERSORT analysis. CPNE8 expression was positively correlated with T regulatory cells and CD56bright cell abundance, and negatively correlated with dendritic cell resting, CD56dim and monocyte in ESCA. CPNE8 expression was positively correlated with the abundance of macrophage, mast cell and NKT cells. The abundance of various immune cells could mediate the progression or regression of tumor cells. Immunotherapies targeting tumor immune microenvironment gain some advancements recently, such as anti-CTLA4, anti-PD-1/PD-L1[26]. The immunotherapies could induce durable anti-tumor immunity, thus making them promising strategies in cancer treatments[27, 28]. Currently, there is still lack of definite biomarker in predicting the responses to immunotherapies. Applications of TMB and MSI could partially predict the possible efficiency of anti-PD1/PD-L1[29, 30]. Here we found that CPNE8 expression was negatively correlated with TMB and MSI status in STAD.CPNE8 expression was positively correlated with TMB in HNSC, and negatively correlated with TMB in THCA. These results might partially explain the response rate of anti-PD1 was much lower in STAD than that in HNSC. And the expression of CPNE8 was much higher in nonresponse group than that in response group according to the IMvigor cohort, which indicating that CPNE8 expression could predict the response of Atezolizumab in advanced UC. However, due to the limited number of clinical trials concerning the immunotherapies in cancer treatment, the potentials of CPNE8 expression in predicting the response to immunotherapies were restrained.

Although CPNE8 failed to demonstrate significant differences between normal tissues and tumor tissues in STAD. We can see that the expression of CPNE8 was higher in tumor tissues. CPNE8 expression was positively correlated with advanced tumor stage, poor OS, DSS, DFS and PFS and TME in STAD, which making it an ideal oncogene in STAD. Thus, we further explored the upstream mechanism that regulate CPNE8 expression in STAD. LncRNAs regulate mRNA expression by sponging miRNA, which is called ceRNA network. The ceRNA network of CPNE8 was not well elucidated. Liu et al found that CPNE8 was a candidate target genes of miR-375 in malignant breast cancer[23]. LncRNA RP11-396F22.1

overexpression predicted poor survival in early-stage cervical cancer. And knockdown of RP11-396F22.1 could upregulate CPNE8 expression to regulate the migration ability of cervical cancer cells[15]. Here, PWAR5 or LIFR-AS1-miR-222-5p-CPNE8 ceRNA network were constructed according to TCGA database and ENCORI database.

The effects of PWAR5 were largely unknown and still controversial. You et al identified PWAR5 as an oncogenic IncRNA in papillary thyroid carcinoma. And low PWAR5 expression was correlated with better OS[31]. Another study in glioma revealed that PWAR5 worked as a tumor suppressor[32]. LIFR-AS1 was identified as an oncogenic IncRNA in some tumors, including pancreatic cancer[33], osteosarcoma[34], 
glioma[35]. Studies in non small cell lung cancer[36] and breast cancer[37] showed that LIFR-AS1 might work as a tumor suppressor. Wang et al found that high LIFR-AS1 expression was correlated with poor overall survival of gastric cancers patients[38]. Pan et al revealed that LIFR-AS1 promoted the proliferation and migration of gastric cancer cells by elevating the expression of COL1A2 through sponging miR-29a-3p[39]. Here, PWAR5 expression or LIFR-AS1 expression was negatively correlated with miR-222-5p and positively correlated with CPNE8, which indicating that PWAR5 or LIFR-AS1 might sponge miR-222-5p to regulate the expression of CPNE8. However, more experiments need to be utilized to verify these results.

Moreover, CPNE8 expression was significantly correlated with the expression of immune cell related genes, such as T cells, B cells, macrophages, neutrophils and dendritic cells. These results strongly indicated that upregulated CPNE8 expression might result in modulating the tumor immune microenvironment. However, there are some limitations of this study, especially the verification in our clinical specimens, cellular functions and mice experiments. It's worth of being further explored.

\section{Conclusion}

In summary, pan cancer analysis revealed that CPNE8 could work as immune related biomarkers in predicting the survival of multiple cancer patients. Moreover, construction of PWAR5 or LIFR-AS1/miR222-5p /CPNE8 ceRNA network further revealed the potential mechanisms of aberrant CPNE8 expression in STAD. These results strongly indicate that CPNE8 is a promising biomarker demonstrating prognostic and immunological roles in cancers.

\section{Abbreviations}

CPNE8, Calcium-dependent protein copine 8; ceRNA, Competitive endogenous RNA; UCSC Xena, University of California Santa Cruz Xena; KM analysis, Kaplan Meier analysis; ICls, Immune checkpoint inhibitors; STAD, Stomach adenocarcinoma; GSEA, Gene set enrichment analysis; TMB, Tumor mutation burden; MSI, Microsatellite instability; TME, Tumor immune microenvironment; ncRNAs, Non-coding RNAs; IncRNA, Long noncoding RNA; miRNA, MicroRNA; TCGA, The Cancer Genome Atlas; OS, Overall survival; DSS, Disease specific survival; DFS, Disease free survival; PFS, Progression free survival; UC, Urothelial carcinoma; LAML, Acute Myeloid Leukemia; LGG, Brain Lower Grade Glioma; CHOL, Cholangiocarcinoma; ESCA, Esophageal carcinoma; HNSC, Head and Neck squamous cell carcinoma; KIRC, Kidney renal clear cell carcinoma; KIRP, Kidney renal papillary cell carcinoma; LUSC, Lung squamous cell carcinoma; BLCA, Bladder Urothelial Carcinoma; BRCA, Breast invasive carcinoma; COAD, Colon adenocarcinoma; KICH, Kidney Chromophobe; PRAD, Prostate adenocarcinoma; READ, Rectum adenocarcinoma; THCA, Thyroid carcinoma; UCEC. Uterine Corpus Endometrial Carcinoma; SKCM, Skin Cutaneous Melanoma; KICH; UCS, Uterine Carcinosarcoma; ACC, adrenocortical carcinoma.

\section{Declarations}




\section{Data Availability}

All data in our study are available from the corresponding authors upon reasonable request.

\section{Conflicts of Interest}

All the authors declare that there are no conflicts of interest.

\section{Funding Statement}

This research was funded by Natural Science Foundation of Guangdong Province, China (No. 2021A1515011113), Guangzhou Science and Technology Program (No. 201904010427), and Guangzhou General Science and technology proj.

\section{Author contributions}

Lin Lu and Xiaofei Cao conceived the project; Guolong Liu supervised this project; Lin Lu and Peiling Zhang conducted data analysis; Lin Lu, Peiling Zhang and Mingmei Guan drafted the manuscript; Qian Peng and Mingmei Guan revised the manuscript. All authors reviewed the manuscript.

\section{References}

1. Chen W, Zheng R, Baade P, Zhang S, Zeng H, Bray F, Jemal A, Yu X, He J: Cancer statistics in China, 2015. CA: a cancer journal for clinicians 2016, 66(2):115-132.

2. Siegel R, Miller K, Jemal A: Cancer statistics, 2018. CA: a cancer journal for clinicians 2018, 68(1):730 .

3. Torre L, Siegel R, Jemal A: Lung Cancer Statistics. Advances in experimental medicine and biology 2016, 893:1-19.

4. Chaft J, Rimner A, Weder W, Azzoli C, Kris M, Cascone T: Evolution of systemic therapy for stages I-III non-metastatic non-small-cell lung cancer. Nature reviews Clinical oncology 2021.

5. Ward Z, Scott A, Hricak H, Atun R: Global costs, health benefits, and economic benefits of scaling up treatment and imaging modalities for survival of 11 cancers: a simulation-based analysis. The Lancet Oncology 2021, 22(3):341-350.

6. Krug K, Jaehnig E, Satpathy S, Blumenberg L, Karpova A, Anurag M, Miles G, Mertins P, Geffen Y, Tang $L$ et al: Proteogenomic Landscape of Breast Cancer Tumorigenesis and Targeted Therapy. Cell 2020, 183(5):1436-1456.e1431.

7. Vogel A, Saborowski A: Medical therapy of HCC. Journal of hepatology 2021.

8. Zhou X, Yao Z, Bai H, Duan J, Wang Z, Wang X, Zhang X, Xu J, Fei K, Zhang Z et al: Treatment-related adverse events of PD-1 and PD-L1 inhibitor-based combination therapies in clinical trials: a systematic review and meta-analysis. The Lancet Oncology 2021. 
9. Wang M, Herbst R, Boshoff C: Toward personalized treatment approaches for non-small-cell lung cancer. Nature medicine 2021.

10. Adusumilli P, Zauderer M, Riviere I, Solomon S, Rusch V, O'Cearbhaill R, Zhu A, Cheema W, Chintala N, Halton $\mathrm{E}$ et al: A phase I trial of regional mesothelin-targeted CAR T-cell therapy in patients with malignant pleural disease, in combination with the anti-PD-1 agent pembrolizumab. Cancer discovery 2021.

11. Nagasawa S, Ikeda K, Horie-Inoue K, Sato S, Itakura A, Takeda S, Hasegawa K, Inoue S: Systematic Identification of Characteristic Genes of Ovarian Clear Cell Carcinoma Compared with High-Grade Serous Carcinoma Based on RNA-Sequencing. International journal of molecular sciences 2019, 20(18).

12. Bu D, Lewis C, Sarode V, Chen M, Ma X, Lazorwitz A, Rao R, Leitch M, Moldrem A, Andrews V et al: Identification of breast cancer DNA methylation markers optimized for fine-needle aspiration samples. Cancer epidemiology, biomarkers \& prevention : a publication of the American Association for Cancer Research, cosponsored by the American Society of Preventive Oncology 2013, 22(12):2212-2221.

13. Maitra R, Grigoryev D, Bera T, Pastan I, Lee B: Cloning, molecular characterization, and expression analysis of Copine 8. Biochemical and biophysical research communications 2003, 303(3):842-847.

14. Dang T, Westcott J, Maine E, Kanchwala M, Xing C, Pearson G: $\Delta$ Np63a induces the expression of FAT2 and Slug to promote tumor invasion. Oncotarget 2016, 7(19):28592-28611.

15. Zhao Y, Huang J, Liu T, He S, Shang C, Guo L, Du Q, Yao S: Overexpression of long non-coding RNA RP11-396F22.1 correlates poor prognosis of patients with early-stage cervical cancer. American journal of translational research 2018, 10(3):684-695.

16. Boudou-Rouquette P, Arrondeau J, Gervais C, Durand J, Fabre E, De Percin S, Villeminey C, Piketty A, Rassy N, Ulmann G et al: Development and validation of a host-dependent, PDL1-independent, biomarker to predict 6-month progression-free survival in metastatic non-small cell lung cancer (mNSCLC) patients treated with anti-PD1 immune checkpoint inhibitors (ICI) in the CERTIM Cohort: The ELY study. EBioMedicine 2021, 73:103630.

17. Espejo-Freire A, Elliott A, Rosenberg A, Costa P, Barreto-Coelho P, Jonczak E, D'Amato G, Subhawong T, Arshad J, Diaz-Perez J et al: Genomic Landscape of Angiosarcoma: A Targeted and Immunotherapy Biomarker Analysis. Cancers 2021, 13(19).

18. Massafra M, Passalacqua M, Gebbia V, Macrì P, Lazzari C, Gregorc V, Buda C, Altavilla G, Santarpia M: Immunotherapeutic Advances for NSCLC. Biologics : targets \& therapy 2021, 15:399-417.

19. Qiu P, Guo Q, Yao Q, Chen J, Lin J: Characterization of Exosome-Related Gene Risk Model to Evaluate the Tumor Immune Microenvironment and Predict Prognosis in Triple-Negative Breast Cancer. Frontiers in immunology 2021, 12:736030.

20. Shadbad M, Asadzadeh Z, Hosseinkhani N, Derakhshani A, Alizadeh N, Brunetti O, Silvestris N, Baradaran B: A Systematic Review of the Tumor-Infiltrating CD8 T-Cells/PD-L1 Axis in High-Grade Glial Tumors: Toward Personalized Immuno-Oncology. Frontiers in immunology 2021, 12:734956. 
21. Winkle M, El-Daly S, Fabbri M, Calin G: Noncoding RNA therapeutics - challenges and potential solutions. Nature reviews Drug discovery 2021, 20(8):629-651.

22. Karreth F, Pandolfi P: ceRNA cross-talk in cancer: when ce-bling rivalries go awry. Cancer discovery 2013, 3(10):1113-1121.

23. Liu J, Wang P, Zhang P, Zhang X, Du H, Liu Q, Huang B, Qian C, Zhang S, Zhu W et al: An integrative bioinformatics analysis identified miR-375 as a candidate key regulator of malignant breast cancer. Journal of applied genetics 2019, 60:335-346.

24. Hu S, Liu H, Zhang J, Li S, Zhou H, Gao Y: Effects and prognostic values of miR-30c-5p target genes in gastric cancer via a comprehensive analysis using bioinformatics. Scientific reports 2021 , 11(1):20584.

25. Reinhardt P, Schmid B, Burbulla L, Schöndorf D, Wagner L, Glatza M, Höing S, Hargus G, Heck S, Dhingra $A$ et al: Genetic correction of a LRRK2 mutation in human iPSCs links parkinsonian neurodegeneration to ERK-dependent changes in gene expression. Cell stem cell 2013, 12(3):354367.

26. Bongiovanni A, Maiorano B, Azzali I, Liverani C, Bocchini M, Fausti V, Di Menna G, Grassi I, Sansovini M, Riva N et al: Activity and Safety of Immune Checkpoint Inhibitors in Neuroendocrine Neoplasms: A Systematic Review and Meta-Analysis. Pharmaceuticals (Basel, Switzerland) 2021, 14(5).

27. Han X, Wei Q, Lv Y, Weng L, Huang H, Wei Q, Li M, Mao Y, Hua D, Cai X et al: Ginseng-derived nanoparticles potentiate immune checkpoint antibody efficacy by reprogramming the cold tumor microenvironment. Molecular therapy : the journal of the American Society of Gene Therapy 2021.

28. Zhou M, Luo C, Zhou Z, Li L, Huang Y: Improving anti-PD-L1 therapy in triple negative breast cancer by polymer-enhanced immunogenic cell death and CXCR4 blockade. Journal of controlled release : official journal of the Controlled Release Society 2021, 334:248-262.

29. Hu X, Xu H, Xue Q, Wen R, Jiao W, Tian K: The role of ERBB4 mutations in the prognosis of advanced non-small cell lung cancer treated with immune checkpoint inhibitors. Molecular medicine (Cambridge, Mass) 2021, 27(1):126.

30. Cho Y, Lee H, Kim D, Kim H, Ha S, Choi Y, Jang K, Kim K: PD-L1 Expression Is Significantly Associated with Tumor Mutation Burden and Microsatellite Instability Score. Cancers 2021, 13(18).

31. You X, Yang S, Sui J, Wu W, Liu T, Xu S, Cheng Y, Kong X, Liang G, Yao Y: Molecular characterization of papillary thyroid carcinoma: a potential three-IncRNA prognostic signature. Cancer management and research 2018, 10:4297-4310.

32. Wang X, Shan C, Deng X, Li L, Ma W: Long non-coding RNA PAR5 inhibits the proliferation and progression of glioma through interaction with EZH2. Oncology reports 2017, 38(5):3177-3186.

33. Chen J, Tao Y, Hong Y, Li H, Huang Z, Xu X, Zheng H, Hu L: MA-mediated up-regulation of LncRNA LIFR-AS1 enhances the progression of pancreatic cancer via miRNA-150-5p/ VEGFA/Akt signaling. Cell cycle (Georgetown, Tex) 2021:1-12.

34. Zhang H, Yu Y, Wang J, Han Y, Ren T, Huang Y, Chen C, Huang Q, Wang W, Niu J et al: Macrophagesderived exosomal IncRNA LIFR-AS1 promotes osteosarcoma cell progression via miR-29a/NFIA axis. 
Cancer cell international 2021, 21(1):192.

35. Ding H, Cui L, Wang C: Long noncoding RNA LIFR-AS1 suppresses proliferation, migration and invasion and promotes apoptosis through modulating miR-4262/NF-KB pathway in glioma. Neurological research 2021, 43(3):210-219.

36. Wang Q, Wu J, Huang H, Jiang Y, Huang Y, Fang H, Zheng G, Zhou X, Wu Y, Lei C et al: IncRNA LIFRAS1 suppresses invasion and metastasis of non-small cell lung cancer via the miR-942-5p/ZNF471 axis. Cancer cell international 2020, 20:180.

37. Xu F, Li H, Hu C: LIFR-AS1 modulates Sufu to inhibit cell proliferation and migration by miR-197-3p in breast cancer. Bioscience reports 2019, 39(7).

38. Wang H, LV J, Li H, Wang W, Lin F: High long non-coding LIFR-AS1 expression correlates with poor survival in gastric carcinoma. European review for medical and pharmacological sciences 2020, 24(10):5378-5384.

39. Pan H, Ding Y, Jiang Y, Wang X, Rao J, Zhang X, Yu H, Hou Q, Li T: LncRNA LIFR-AS1 promotes proliferation and invasion of gastric cancer cell via miR-29a-3p/COL1A2 axis. Cancer cell international 2021, 21(1):7.

\section{Tables}

Table 1. Cox regression analysis of CPNE8 for OS in TCGA pan cancer

$\begin{array}{lllll}\text { Cancer } & \text { HR } & \text { HR.95L } & \text { HR.95H } & \text { P value } \\ \text { LGG } & 1.713587852 & 1.342568459 & 2.187138621 & 1.52 \mathrm{E}-05 \\ \text { STAD } & 1.722719794 & 1.319868636 & 2.248529443 & 6.28 \mathrm{E}-05 \\ \text { LAML } & 1.331482633 & 1.153491387 & 1.536939089 & 9.22 \mathrm{E}-05 \\ \text { UCEC } & 1.34190367 & 1.07765066 & 1.670954722 & 0.008580974 \\ \text { BLCA } & 1.205286267 & 1.034640494 & 1.404077063 & 0.016522425 \\ \text { PAAD } & 1.587681987 & 1.063591478 & 2.370020955 & 0.023723375 \\ \text { UCS } & 0.610327128 & 0.385985412 & 0.965060314 & 0.034678351 \\ \text { HNSC } & 1.20193357 & 1.009127239 & 1.431577952 & 0.039225517\end{array}$


Table 2. Cox regression analysis of CPNE8 for DSS in TCGA pan cancer

$\begin{array}{lllll}\text { Cancer } & \text { HR } & \text { HR.95L } & \text { HR.95H } & \text { P value } \\ \text { STAD } & 2.020836094 & 1.4337022 & 2.848414767 & 5.89 \mathrm{E}-05 \\ \text { UCEC } & 1.624146503 & 1.27656136 & 2.066372951 & 7.91 \mathrm{E}-05 \\ \text { LGG } & 1.702345809 & 1.306642767 & 2.217883363 & 8.10 \mathrm{E}-05 \\ \text { BLCA } & 1.26196571 & 1.051433135 & 1.514654048 & 0.012470359 \\ \text { KIRC } & 0.719996404 & 0.555770897 & 0.932749132 & 0.012881653 \\ \text { UCS } & 0.591172883 & 0.364573981 & 0.958613053 & 0.033060503\end{array}$

Table 3. Cox regression analysis of CPNE8 for DFS in TCGA pan cancer

$\begin{array}{lllll}\text { Cancer } & \text { HR } & \text { HR.95L } & \text { HR.95H } & \text { P value } \\ \text { STAD } & 2.395500065 & 1.401209411 & 4.095334012 & 0.001408552 \\ \text { ESCA } & 2.080038245 & 1.203590939 & 3.594708933 & 0.008694047\end{array}$

Table 4. Cox regression analysis of CPNE8 for PFS in TCGA pan cancer 


$\begin{array}{lllll}\text { Cancer } & \text { HR } & \text { HR.95L } & \text { HR.95H } & \text { P value } \\ \text { STAD } & 1.798765284 & 1.353947739 & 2.38972041 & 5.11 \mathrm{E}-05 \\ \text { LGG } & 1.365264309 & 1.108459784 & 1.681564508 & 0.00340601 \\ \text { GBM } & 1.264799185 & 1.030855966 & 1.551833652 & 0.024373618 \\ \text { BLCA } & 1.192227436 & 1.019896569 & 1.393676871 & 0.02729353 \\ \text { KIRP } & 0.636795151 & 0.420739399 & 0.96379865 & 0.032814546 \\ \text { THCA } & 0.472851904 & 0.232229827 & 0.962791584 & 0.038971972\end{array}$

Figures 

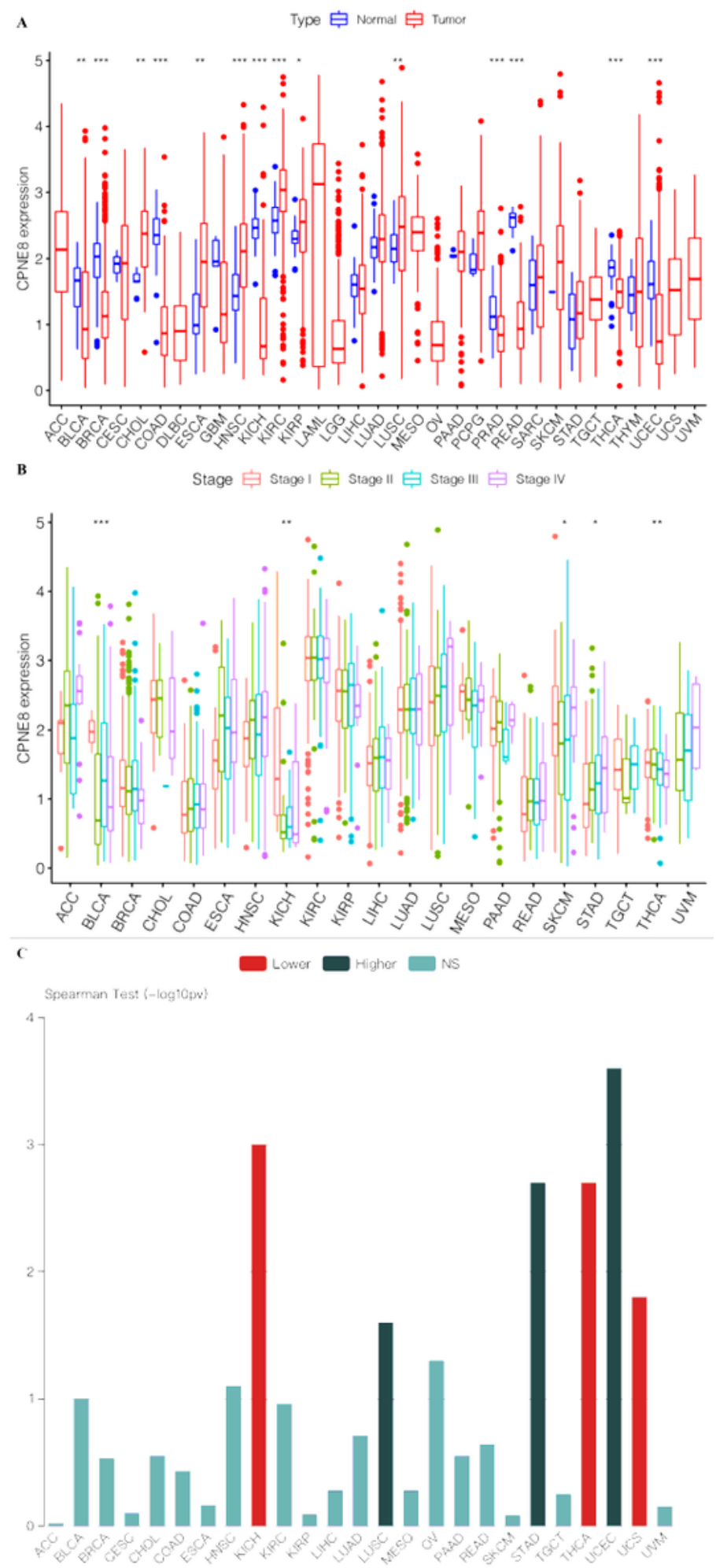

Figure 1

\section{Figure 1}

Expression and significance of CPNE8 in pan cancer. (A) Differential CPNE8 expression were compared between 33 kinds of tumor cohorts and corresponding normal tissues. (B) Correlations between CPNE8 expression and tumor stages in pan cancer were analyzed using data generated from UCSC Xena database. (C) Correlation between CPNE8 expression and tumor stage was analyzed using online tool TISIDB. 

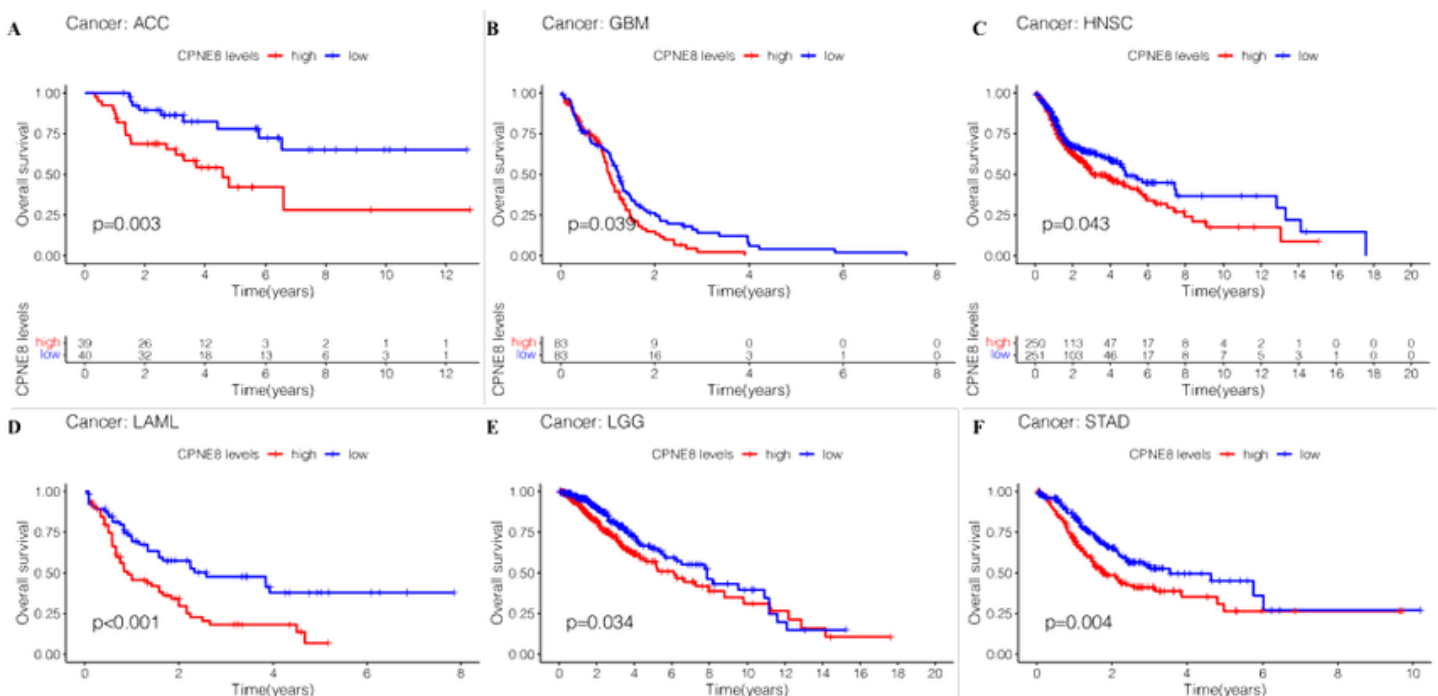

E Cancer: LGG

F Cancer: STAD
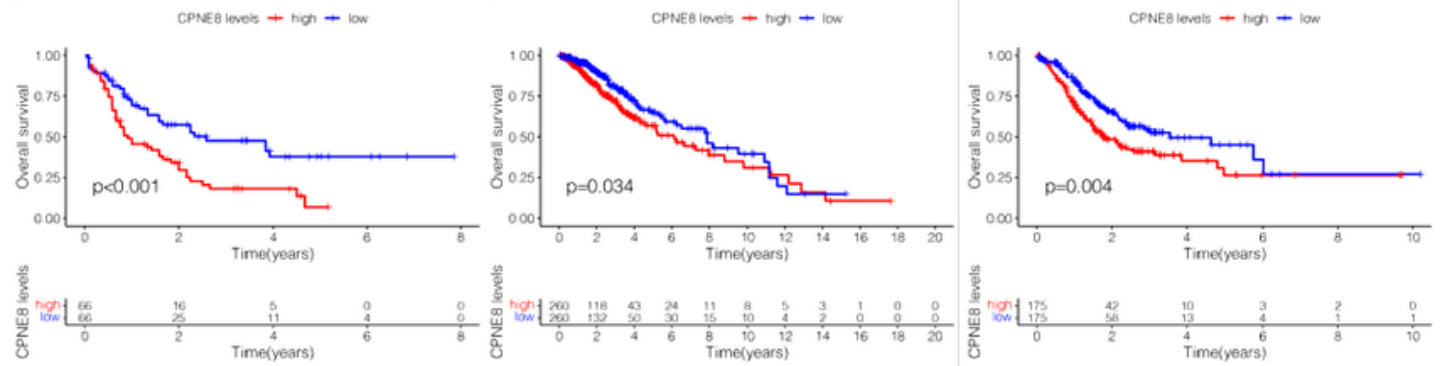

G Cancer: $A C C$

H Canoer: GBM
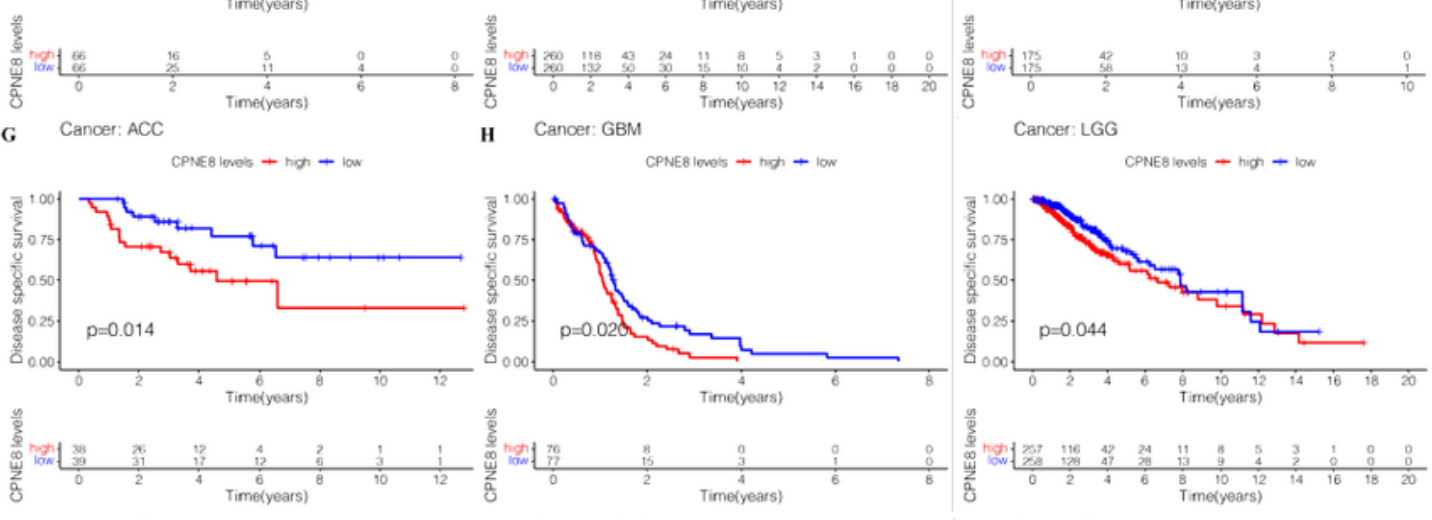

Cancer: LGG
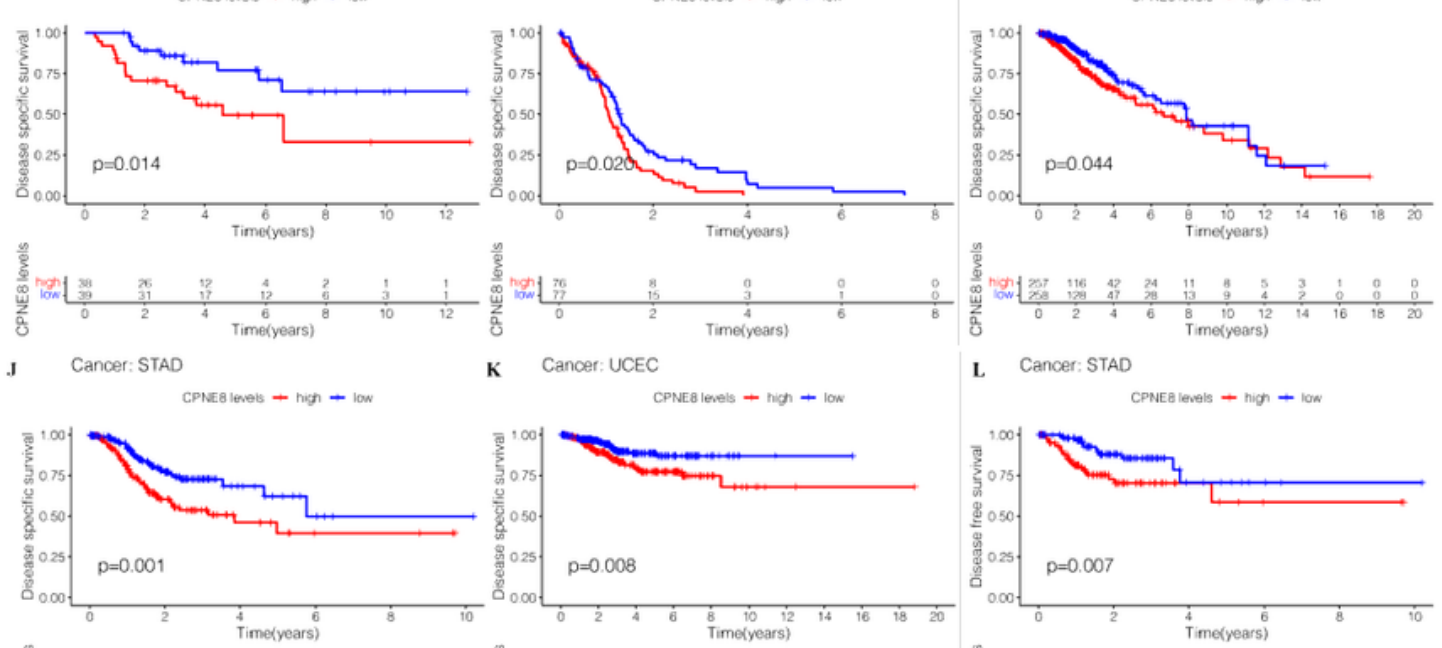

K Cancer: UCEC

L Cancer: STAD
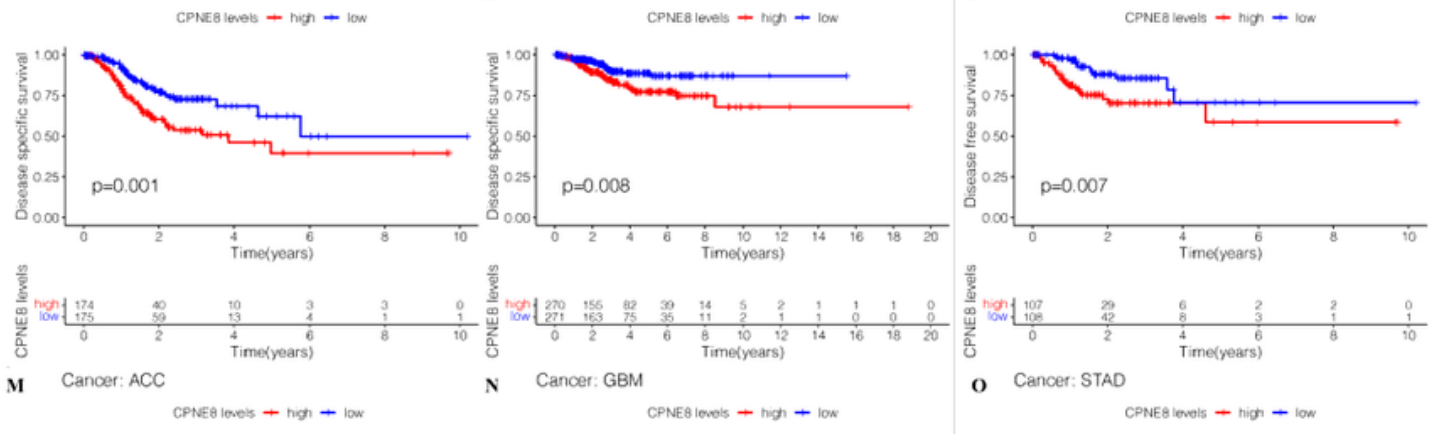

M Cancer: ACC
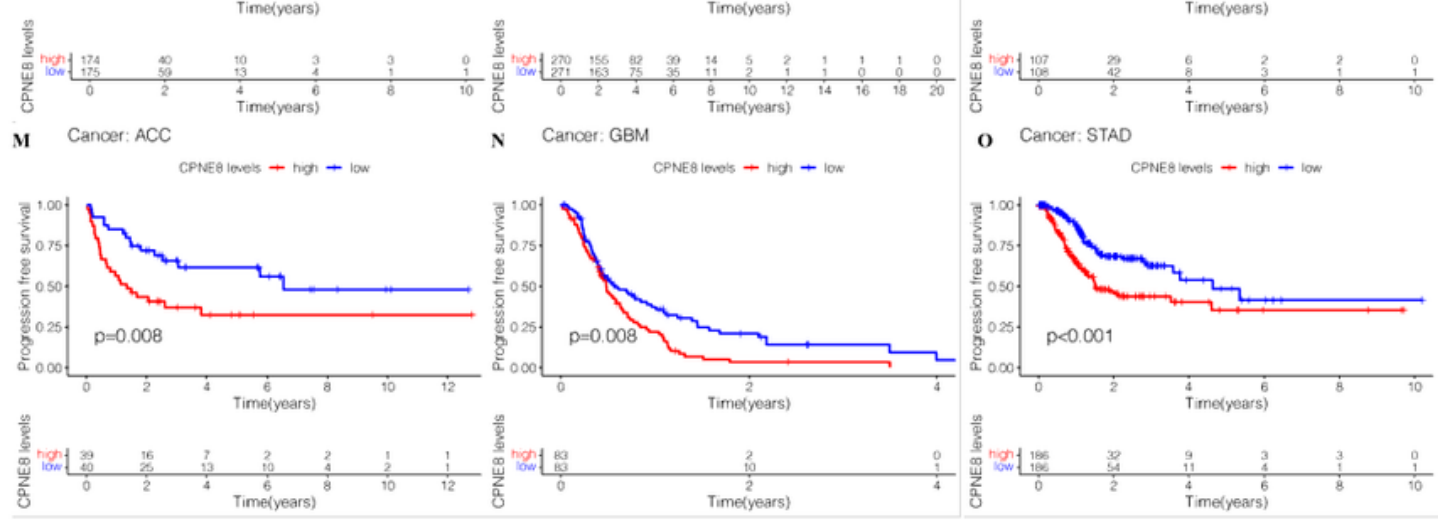

Figure ?

\section{Figure 2}

Prognostic value of CPNE8 in pan cancer. Kaplan-Meier survival curve analysis was performed to investigate the correlations between CPNE8 expression and prognosis of cancer patients. The prognostic roles of CPNE8 in predicting the OS of (A) ACC, (B) GBM, (C) HNSC, (D) LAML, (E) LGG and (F) STAD. KM curves of CPNE8 expression in predicting DSS in (G) ACC, (H) GBM, (I) LGG, (J) STAD and (K) UCEC. KM 
curves of CPNE8 expression in predicting DFS in (L) STAD. KM curves showed the significance of CPNE8 expression in predicting PFS in (M) ACC, (N) GBM, and (O) STAD.
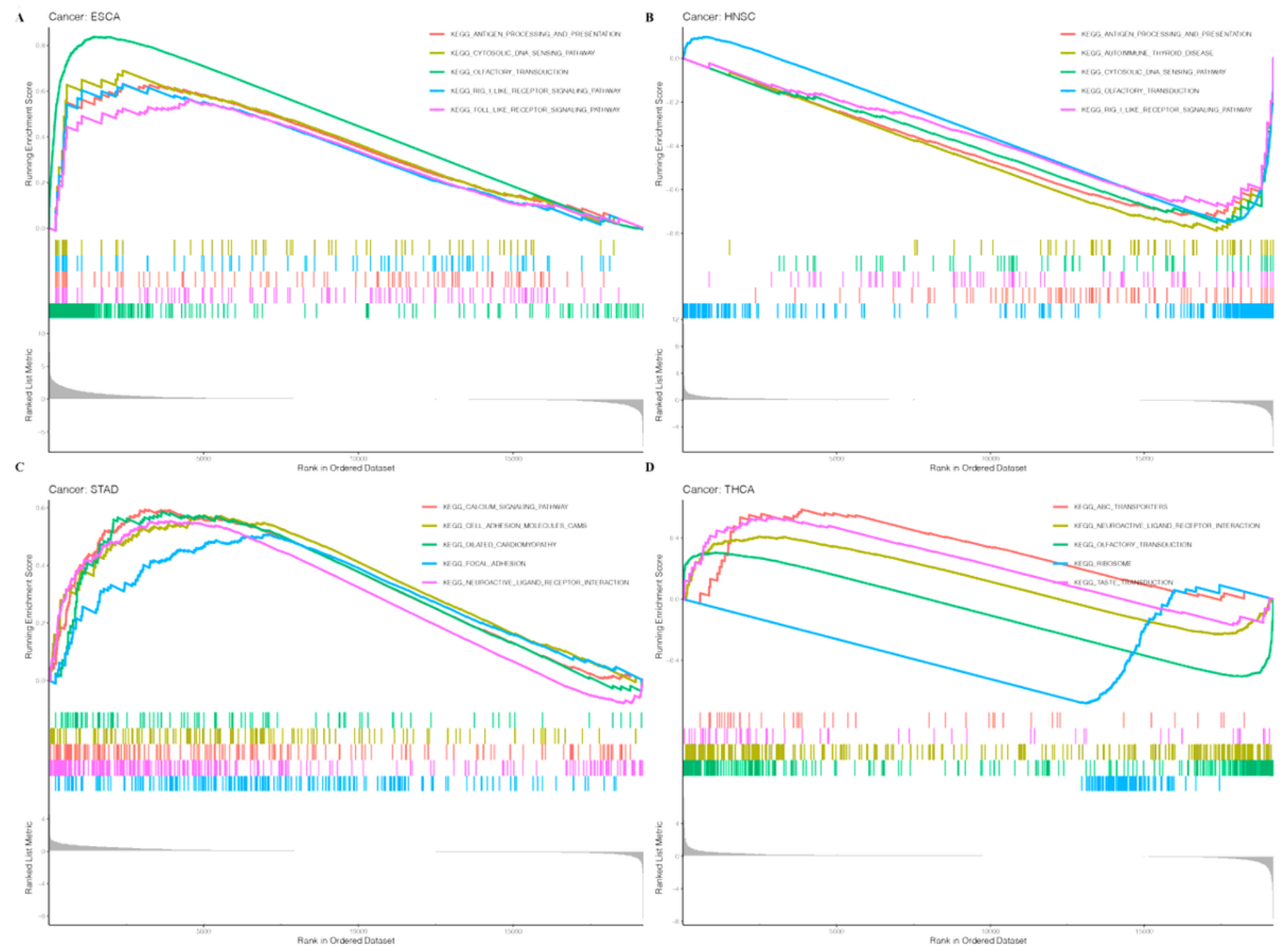

Figure 3

\section{Figure 3}

Functional annotation of CPNE8. GSEA was utilized to study the enrichment of signaling pathways in (A) ESCA, (B) HNSC, (C) STAD and (D) THCA.

\section{Figure 4}

Correlations between CPNE8 expression and immune microenvironment. (A-C) ESTIMATE algorithm and CIBERSORT analysis were performed based on data downloaded from UCSC Xena database. (A) Correlation between CPNE8 expression and stomal score in STAD. Correlation between CPNE8 expression and infiltration of dendritic cells resting (B) and Tregs (C) in ESCA. (D-J) Correlations between CPNE8 expression and abundance of immune cells were analyzed using TISIDB. Correlations between CPNE8 expression and abundance of CD56bright (D), CD56dim (E) and monocyte (F) in ESCA. Associations 
between CPNE8 expression and abundance of macrophages (G), mast cells (I) and NKT cells (I) in STAD. (J) Correlation between CPNE8 expression and abundance of CD56dim in THCA.

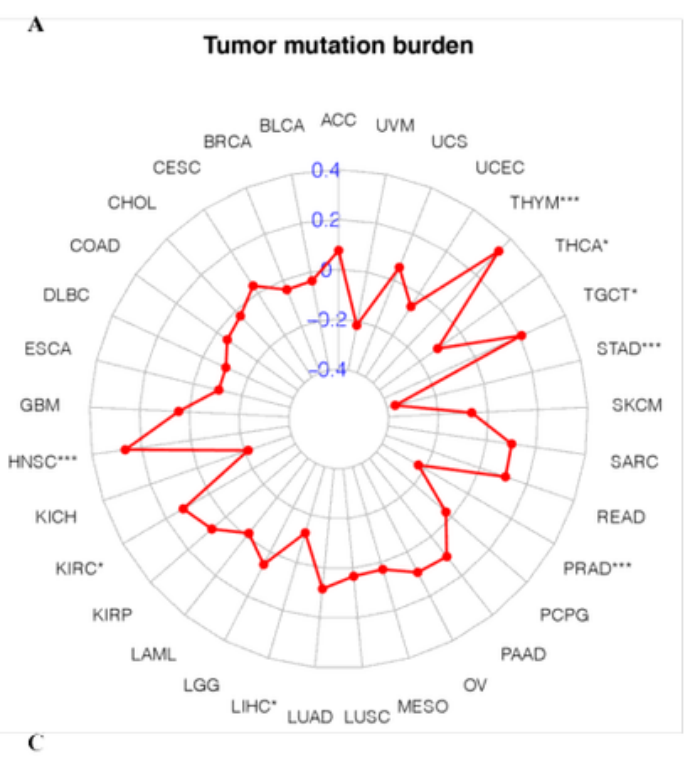

B

Microsatellite instability

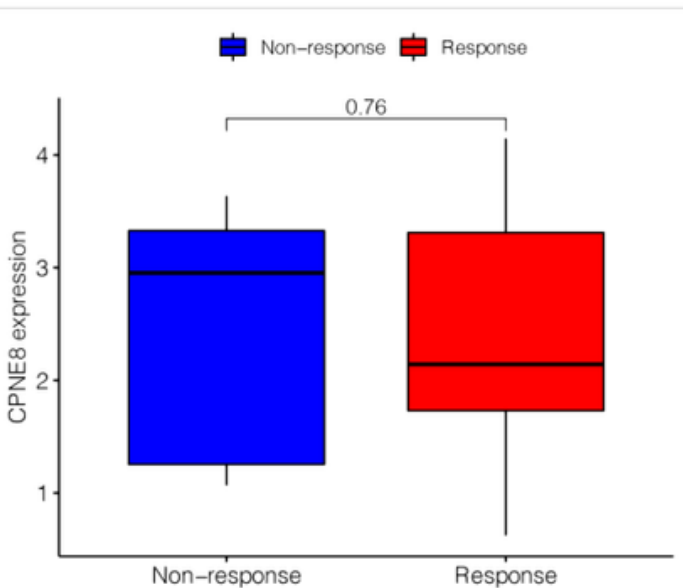

D
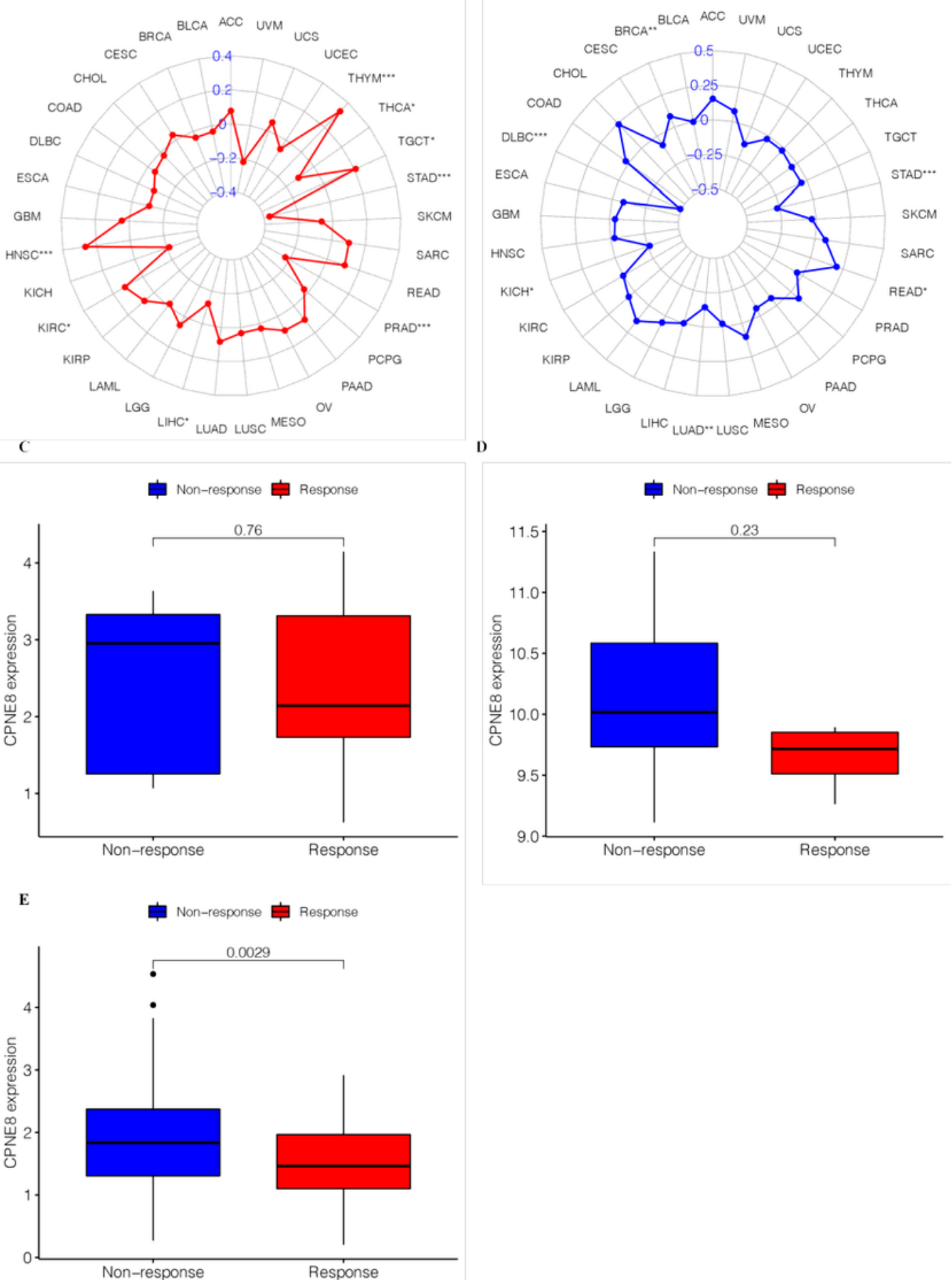

Figure 5

Figure 5

Significance of CPNE8 in predicting immuotherapy response. (A) Correlations between CPNE8 expression and TMB in pan cancer. (B) Correlations between CPNE8 expression and MSI in pan cancer. (B) Differential expression of CPNE8 in ICl response group and non response group in melanoma. (C) 
Differential CPNE8 expression between response group and non response group in renal cell carcinoma. (D) Differential CPNE9 expression between ICI response and nonresponse group in mUC.

\section{Figure 6}

Identification of miRNA could interact with CPNE8 in STAD. (A) miRNAs that could interacted with CPNE8 were analyzed by TargetScan. (B) miR-30e-5p was negatively correlated with CPNE8 expression. (C) Survival analysis of miRNA-30e-5p and CPNE8 in STAD. (D) Potential binding site between CPNE8 and miR-30e-5p. (E) miR-222-3p was negatively correlated with CPNE8 expression. (F) Survival analysis of miR-222-3p plus CPNE8 in STAD. (G) Potential binding site between CPNE8 and miR-222-3p.

\section{Figure 7}

Construction of ceRNA network in STAD. (A) NORAD was positively correlated with CPNE8 expression. (B) NORAD was negatively correlated with miR-30e-5p expression. (C) LIFR-AS1 expression was positively correlated with CPNE8 expression. (D) LIFR-AS1 expression was negatively correlated with miR-222-3p expression. (E) PWAR5 expression was positively correlated with CPNE8 expression. (D) PWAR5 expression was negatively correlated with miR-222-3p expression. (G) Visualization of the ceRNA network.

\section{Figure 8}

Correlations between CPNE8 expression and immune cell related genes in STAD. Correlations between CPNE8 expression and expression of (A) CD4 (B) IRF5 (C) PTGS2 (D) CD163 (E) VSIG4 (F) MS4A4A (G) ITGAM (H) CCR7 (I) CD1C and (J) ITGAX in STAD.

\section{Supplementary Files}

This is a list of supplementary files associated with this preprint. Click to download.

- spFigure1.tif

- spFigure2.tif 